\title{
Current Concepts in the Diagnosis and Management of Pulmonary Embolism
}

\author{
Sridhar Kasturi \\ Sunshine Heart Institute, Secunderabad, Telangana, Hyderabad, India \\ Email: sridharkasturi@yahoo.com
}

How to cite this paper: Kasturi, S. (2021) Current Concepts in the Diagnosis and Management of Pulmonary Embolism. International Journal of Clinical Medicine, 12, 115-129.

https://doi.org/10.4236/ijcm.2021.123013

Received: December 22, 2020

Accepted: March 27, 2021

Published: March 30, 2021

Copyright $\odot 2021$ by author(s) and Scientific Research Publishing Inc. This work is licensed under the Creative Commons Attribution International License (CC BY 4.0).

http://creativecommons.org/licenses/by/4.0/

(c) (i) Open Access

\begin{abstract}
Acute pulmonary embolism (PE) is one of the most common causes of cardiovascular death. Most often acute PE is associated with under diagnosis, misdiagnosis and delay in diagnosis and management leading to high morbidity and mortality. PE outcomes will improve with proper evaluation of clinical symptoms and signs, relevant diagnostic tests, identifying high-risk patients suitable for early re-perfusion with I.V. or catheter-directed thrombolytic therapy or surgical embolectomy and in some cases additional use of mechanical circulatory support. During clinical evaluation modified Geneva score, Well's score, and Simplified pulmonary embolism severity index (sPESI) scores are useful in assessing PE and its adverse outcomes. Hestia criteria are useful in identifying suitable for outpatient management of PE. Long-term management of PE involves identifying patients prone for recurrence and CTPE with appropriate long-term prophylaxis using oral anticoagulants.
\end{abstract}

\section{Keywords}

Pulmonary Embolism, Management of PE

\section{Introduction}

Acute pulmonary embolism (PE) is due to Deep Vein Thrombosis (DVT) embolism, i.e. blood clot or part of it breaks off from the vein. DVT is a formation of thrombosis in the deep vein of the lower extremity or pelvis either partially or blocking blood flow [1]. Acute PE ranges from asymptomatic, incidentally discovered sub-segmental thrombi to massive PE complicated by cardiogenic shock and multi-organ dysfunction. The most common PE source is lower extremity sites and unusual sites are the right heart, upper extremity, renal veins, iliac veins and hepatic veins.

Sudden occlusion of the pulmonary artery and its branches results in abrupt 
increase of pulmonary vascular resistance to a level of afterload that cannot be matched by the RV causing RV dysfunction, failure, and sometimes sudden death due to Electro-mechanical dissociation. Clinical symptoms and signs of PE depend on the extent and duration of the pulmonary artery obstruction and pre-existing cardiopulmonary status.

\section{Epidemiology}

$\mathrm{PE}$ is the $3^{\text {rd }}$ most common cause of cardiovascular death [2]. It constitutes approximately 100,000 to 180,000 deaths/annum in USA. Vein thrombus-embolism (VTE) mortality was observed in more than 370,000 people of 6 European countries annually, which is more than the reported combined mortality from AIDS, breast cancer, prostate cancer and traffic accidents. Clinical spectrum of PE varies from asymptomatic to complete cardiovascular collapse. Autopsy based study of 1000 patients observed $15.9 \%$ was the reported incidence of PE in India. $\mathrm{PE}$ is underestimated, underdiagnosed and undertreated in most patients, and it was estimated that only $8 \%$ of patients were exactly diagnosed as a case of PE before death. Untreated DVT/PE has serious long term consequences like recurrent VTE, post-thrombotic syndrome, venous ulceration and chronic thromboembolic pulmonary hypertension (Figure 1). Every effort should be made to clear the clot as early as possible to improve acute hemodynamic status by reversing acute and sub-acute RV dysfunction, chronic thrombotic pulmonary embolism (CTPE), and lowering the mortality rate. CT-PE is a serious complication of PE occurs in $4 \%$ of patients and if untreated, associated with a $90 \%$ mortality rate.

\section{Classification, Assessment of Clinical (Pre-Test) Probability and Risk stratification}

$\mathrm{PE}$ patients can be categorized as low-risk $\mathrm{PE}$, sub-massive $\mathrm{PE}$, and massive $\mathrm{PE}$. Low-risk PE constitutes 55\% of PE patients carries a good prognosis with a

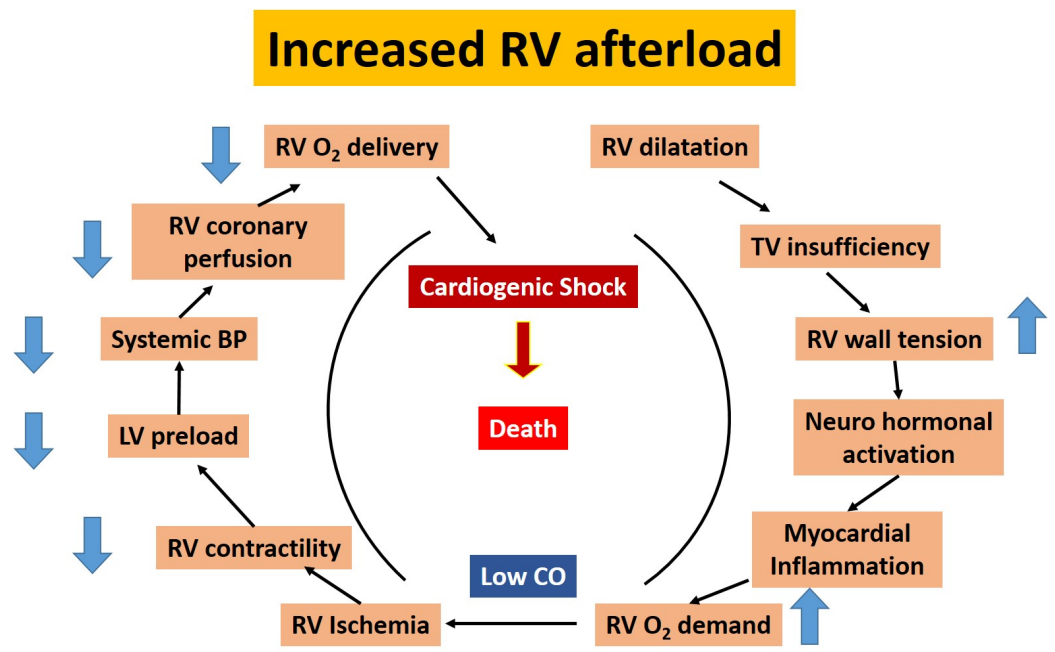

Figure 1. Key factors contributing to hemodynamic collapse and death in acute pulmonary embolism. 
mortality of $1 \%$, sub-massive PE was observed in $40 \%$ of patients with a mortality of $21 \%$ at three months. In contrast, massive PE was observed on average of $5 \%$ of PE patients with $58 \%$ mortality at three months [3].

Pulmonary embolism can be predicted clinically using wells score and revised Geneva score in a clinically suspected PE case to plan appropriate strategies for treating PE (Table 1).

The spiral of hemodynamic collapse in acute PE (Figure 1): ESC 2019 PE guidelines.

\section{Risk assessment of PE:}

PE patients can be classified (Table 2) as a high-risk, intermediate-risk and low-risk group. High-risk group patients are with hemodynamic instability in contrast to the other two groups. Low-risk patients are without elevated Troponin-I, RV dysfunction, and PESI III to IV or SPESI $\geq 1$. Intermediate risk group patients have increased troponin I or RV dysfunction with PESI III to IV or SPESI $\geq 1$.

Table 3: Simplified pulmonary embolism severity index (PESI) [4].

Patients with none of the clinical variable (i.e., the total score of 0 ) are considered as low risk and have mortality and pulmonary embolism-related complication rates significantly lower as those with a score of $\geq 1$.

Table 1. Pulmonary embolism can be predicted clinically using wells score and revised Geneva score.

\begin{tabular}{|c|c|c|c|}
\hline Revised Geneva Score & & Wells Score & \\
\hline Variable & Points & Variable & Points \\
\hline Predisposing factors & & Predisposing factors & \\
\hline Age $>65$ years & +1 & & \\
\hline Previous DVT or PE & +3 & Previous DVT or PE & +1.5 \\
\hline Surgery or fracture within one month & +2 & Recent surgery or immobilization & +1.5 \\
\hline Active malignancy & +2 & Cancer & +1 \\
\hline Symptoms & & Symptoms & \\
\hline Unilateral lower limb pain & +3 & & \\
\hline Hemoptysis & +2 & Hemoptysis & +1 \\
\hline Clinical Signs & & Clinical Signs & \\
\hline Heart rate & & Heart rate & \\
\hline $\begin{array}{c}75-94 \text { beats } / \mathrm{min} \\
\pm 95 \text { beats } / \mathrm{min}\end{array}$ & +3 & $>100$ beats/min & +1.5 \\
\hline $\begin{array}{l}\text { Pain on lower limb deep vein at } \\
\text { palpitation and unilateral edema }\end{array}$ & $\begin{array}{l}+5 \\
+4\end{array}$ & Clinical signs of DVT & +3 \\
\hline & & Clinical Judgement & \\
\hline & & Alternative diagnosis less likely than PE & +3 \\
\hline Clinical Probability & Total & Clinical Probability (3 levels) & Total \\
\hline Low & $0-3$ & Low & $0-1$ \\
\hline Intermediate & $4-10$ & Intermediate & $2-6$ \\
\hline \multirow[t]{4}{*}{ High } & \pm 11 & High & \pm 7 \\
\hline & & Clinical Probability (2 levels) & Total \\
\hline & & PE unlikely & $0-4$ \\
\hline & & PE likely & $>4$ \\
\hline
\end{tabular}


Table 2. Classification of PE by American heart association (2011) and European society of cardiology (2019).

\begin{tabular}{|c|c|}
\hline AHA CLASSIFICATION (2011) & ESC CLASSIFICATION (2019)* \\
\hline MASSIVE PE & HIGH-RISK PE \\
\hline $\begin{array}{l}\text { Hypotension, defined as a systolic blood pressure } \\
<90 \mathrm{~mm} \mathrm{Hg} \text {, a drop of }>40 \mathrm{~mm} \mathrm{Hg} \text { for at least } 15 \\
\text { minutes, or need for vasopressor support }\end{array}$ & $\begin{array}{l}\text { One of the following clinical presentations: } \\
\text { cardiac arrest, obstructive shock, persistent } \\
\text { hypotension (systolic BP }<90 \mathrm{mmHg} \text { or a } \\
\text { systolic BP drop >_40 } \mathrm{mmHg} \text { for }>15 \mathrm{~min}\end{array}$ \\
\hline SUBMASSIVE PE & INTERMEDIATE RISK PE \\
\hline $\begin{array}{l}\text { Acute PE without systemic hypotension (systolic } \\
\text { blood pressure }>90 \mathrm{~mm} \mathrm{Hg} \text { ) but with either RV } \\
\text { dysfunction or myocardial necrosis }{ }^{3}\end{array}$ & $\begin{array}{l}\text { Acute PE without systemic hypotension (systolic } \\
\text { blood pressure }>90 \mathrm{~mm} \mathrm{Hg} \text { ) but with either RV } \\
\text { dysfunction or elevated cardiac troponin levels }{ }^{3} \\
\text { Additionally, include PESI/sPESI criteria. }\end{array}$ \\
\hline LOW-RISK PE & LOW-RISK PE \\
\hline $\begin{array}{l}\text { Acute } \mathrm{PE} \text { and the absence of the clinical markers } \\
\text { of adverse prognosis that define massive or } \\
\text { sub-massive } \mathrm{PE}^{3}\end{array}$ & $\begin{array}{l}\text { No RV dysfunction/no elevated cardiac } \\
\text { troponins/normal PESI }\end{array}$ \\
\hline
\end{tabular}

Table 3. Simplified pulmonary embolism severity index.

$\begin{array}{cc}\text { Variable } & \text { Points } \\ \text { Age }>80 \text { years } & 1 \\ \text { History of cancer } & 1 \\ \text { History of heart failure or chronic lung disease } & 1 \\ \text { The pulse rate of } \geq 110 \mathrm{bpm} & 1 \\ \text { Systolic blood pressure }<100 \mathrm{mmHg} & 1 \\ \text { Oxygen saturation }<90 \% \text { on room air } & 1\end{array}$

\section{Diagnosis and Management of PE}

Management of PE depends upon early diagnosis and assessing risk status to decide appropriate re-perfusion and anticoagulant therapy on fast track basis to reduce mortality and morbidity associated with misdiagnosis and delay in the treatment of PE (Figure 2).

\subsection{Plasma D-Dimer}

Plasma D-Dimer is the degradation product of cross-linked fibrin. ELISA derived assays have the highest sensitivity, its measurement recommended in out-patient/emergency department patients with low or intermediate clinical probability, or PE unlikely to reduce the need for unnecessary imaging and irradiation.

\subsection{Troponin}

Elevated plasma troponin (Trop-T/I) concentrations on admission may be associated with a worse prognosis in the acute phase of PE. Elevated Trop-T/I levels 


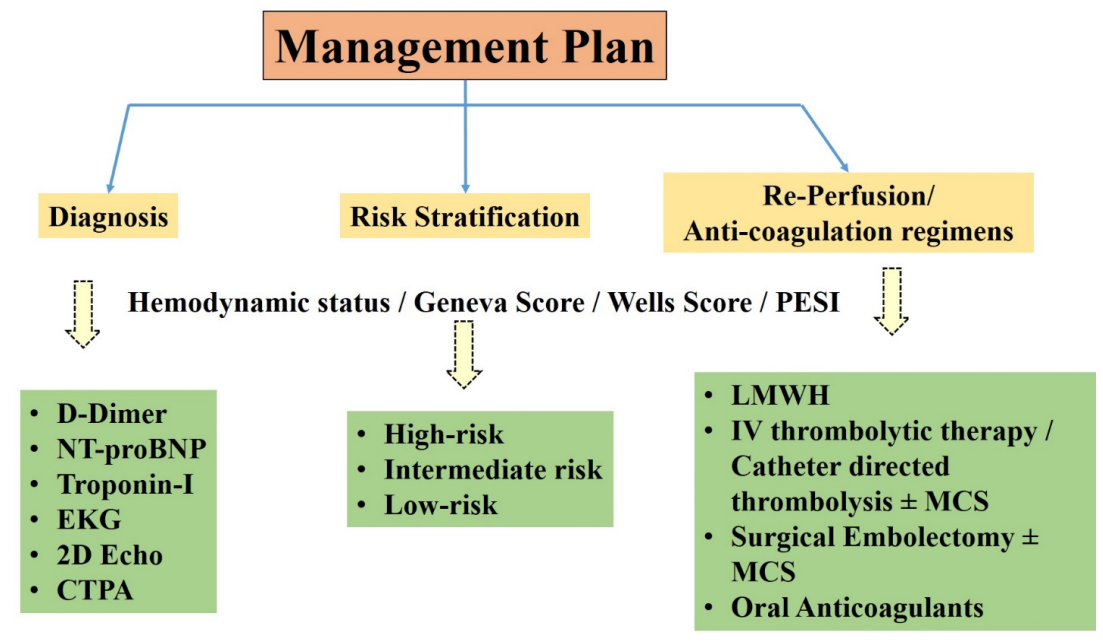

Figure 2. Management of PE.

are observed in approximately $50 \%$ of the patients with acute PE, associated with an increased risk of death and significant adverse events in the acute phase. A meta-analysis showed that elevated troponin concentrations were associated with an increased risk of mortality, both in unselected patients (OR 5.2, 95\% CI 3.3 - 8.4) and in those who were hemodynamically stable at presentation (OR $5.9,95 \%$ CI 2.7 - 13.0) [5].

\subsection{BNP and proBNP}

Elevated BNP or NT-proBNP levels associated with low specificity and positive predictive value for increased mortality rate in hemodynamically stable patients with PE, but low levels of BNP or NT-proBNP will exclude an unfavorable early clinical outcome, with high sensitivity and a negative predictive value. A meta-analysis of 13 studies showed $51 \%$ of 1132 patients with acute PE with elevated brain natriuretic peptide (BNP) or N-terminal (NT)-proBNP levels contributed to high risk of early death and a complicated in-hospital Course [5].

\subsection{EKG}

EKG findings vary from patient to patient. Characteristic findings are-sinus tachycardia, T-wave changes, ST-segment changes, right axis deviation, S1-Q3T3, RBBB and P-pulmonale.

\subsection{Chest X-Ray (Figure 3)}

Primarily to exclude other diagnoses like pneumonia, pneumothorax, CHF, tumour, and rib fracture, which are also helpful in interpreting the V/Q scan. PE can present with radio-graphical signs on chest X-ray like

(https://radiopaedia.org/articles/pulmonary-embolism)-Fleischner sign: Enlarged pulmonary artery, Hampton's Hump: peripheral wedge of airspace opacity and implies lung infarction, Westermark sign: regional oligemia and highest positive predictive value, Knuckle sign: abrupt tapering or cutoff of a pulmonary 


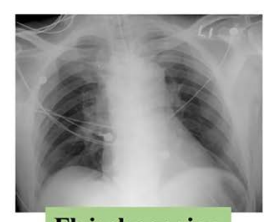

Fleischner sign

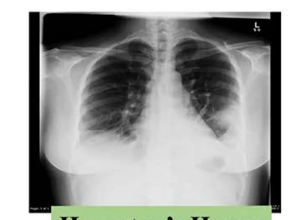

Hampton's Hump

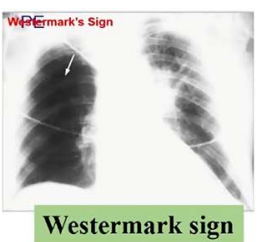

Westermark sign

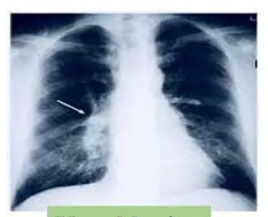

Knuckle sign

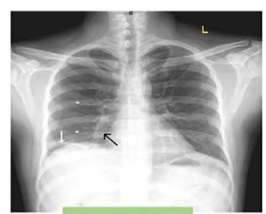

Palla Sign

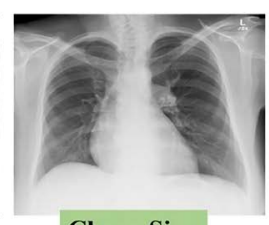

Chang Sign

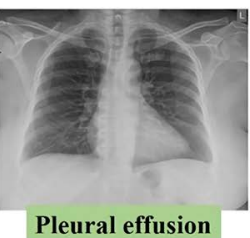

Pleural effusion

Figure 3. Different radio-graphical signs on chest X-ray of PE (https://radiopaedia.org/articles/pulmonary-embolism).

artery secondary to an embolus, Palla Sign: enlarged right descending pulmonary artery, Chang Sign: dilated right descending pulmonary artery with sudden cut-off, and in some cases Pleural effusion evident (35\%).

\subsection{Echocardiogram}

Transesophageal Echocardiography (TEE) more sensitive than Transthoracic Echocardiography (TTE). It may demonstrate intra-cardiac, main pulmonary artery, and its main branches clots, RV enlargement and signs of right ventricular dysfunction. TTE should be performed to all patients with PE, and feasible in patients with stable hemodynamic status (Figure 4(a) \& Figure 4(b)).

Echocardiographic RV/LV ratio $\geq 0.9$ shown to be an independent predictor of hospital mortality. Registry of 1416 patients showed mortality rate is $1.9 \%$ if the $\mathrm{RV} / \mathrm{LV}$ ratio is of $<0.9$, and $6.6 \%$ if the $\mathrm{RV} / \mathrm{LV}$ ratio is of $\geq 0.9$. Retrospective analysis of 120 patients with hemodynamically stable PE based on chest CT showed mortality at 3 months: $17 \%$ if $\mathrm{RV} / \mathrm{LV} \geq 1.5,8 \%$ if $1 \leq \mathrm{RV} / \mathrm{LV}<1.5$ and $0 \%$ if RV/LV $1.0[6]$.

\subsection{Computed Tomography Pulmonary Angiography (CTPA)}

It has an excellent accuracy in diagnosing $\mathrm{PE}$ and may provide alternative diagnoses if PE is excluded with short acquisition time. Main disadvantages of CTPA are exposure to radiation and contrast related problems. CT identifies proximal $\mathrm{PE}$, which is more often associated with the hemodynamic imbalance and not very accurate in diagnosing peripheral $\mathrm{PE}$. CT can show enlarged right ventricle and estimation of the right ventricle and left ventricle ratio (Figure 5).

$\mathrm{CT}$ scan showing right pulmonary thrombus with RV enlargement (RV/LV ratio $>1$ ) suggestive of massive pulmonary embolism (PE)

\subsection{Planar V/Q Scan}

Is useful in diagnosing $\mathrm{PE}$ but available in limited centres, relatively expensive, no contraindications, inconclusive in $50 \%$ cases, and cannot provide the alternative diagnosis. 

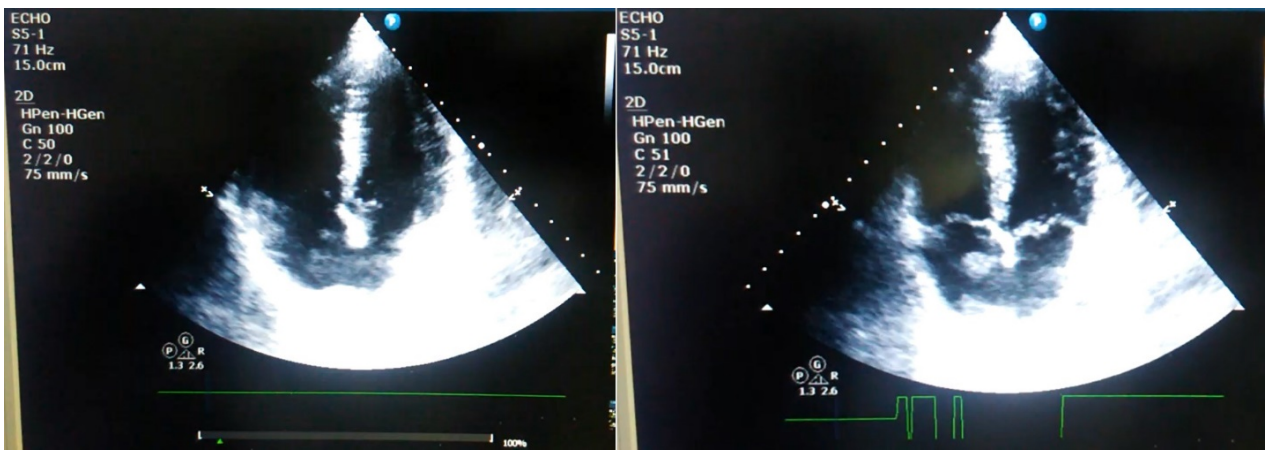

(a)

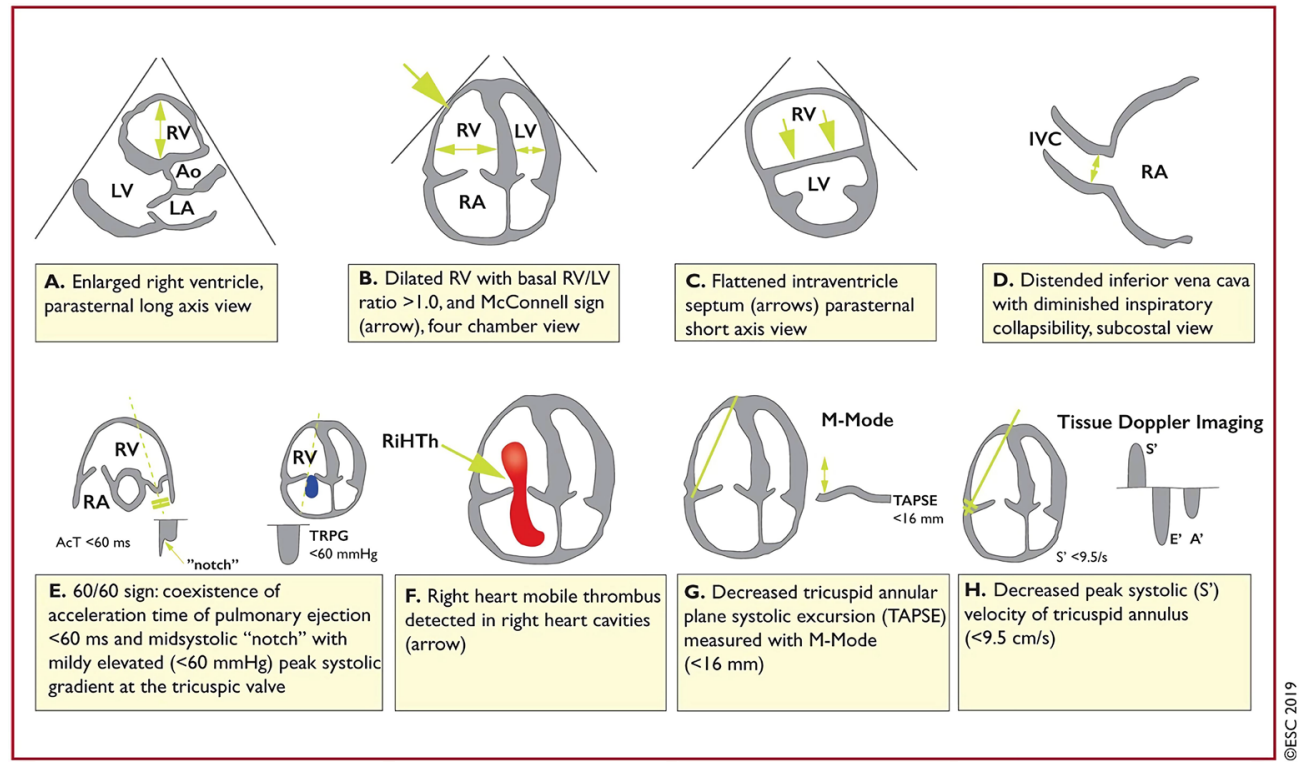

(b)

Figure 4. (a) Trans-esophageal Echocardiography (TEE), Trans-thoracic Echocardiography (TTE); (b) Graphic representation of transthoracic echocardiographic parameters in the assessment of right ventricular pressure overload (ESC-2019). A' = peak late diastolic (during atrial contraction) velocity of tricuspid annulus by tissue Doppler imaging; AcT = right ventricular outflow Doppler acceleration time; Ao = aorta; E' = peak early diastolic velocity of tricuspid annulus by tissue Doppler imaging; IVC = inferior vena cava; LA = left atrium; LV = left ventricle; RA = right atrium; RiHTh = right heart thrombus (or thrombi); RV = right ventricle/ventricular; $\mathrm{S}^{\prime}$ = peak systolic velocity of tricuspid annulus by tissue Doppler imaging; TAPSE = tricuspid annular plane systolic excursion; TRPG = tricuspid valve peak systolic gradient.

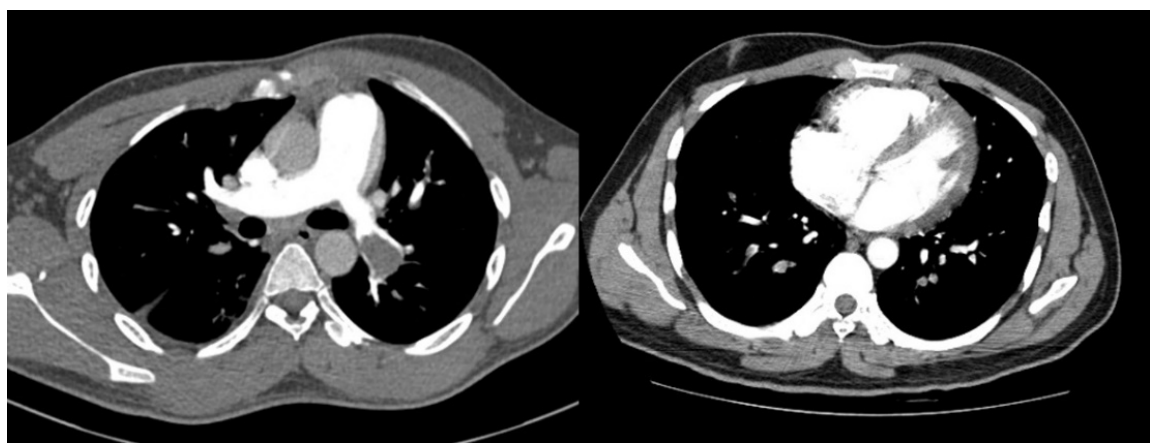

Figure 5. Computed tomography pulmonary angiography for PE. 


\subsection{Compression Ultrasonography Lower Limb (CUS)}

If CUS shows proximal DVT in a patient with clinical suspicion of PE, PE diagnosis is acceptable, and if it shows only a distal DVT, further testing should be considered to confirm PE.

\subsection{Pulmonary Angiogram (PA)}

Most specific test, can detect even small emboli, presents like intraluminal filling defects, and vascular cutoffs. It is associated with a $0.5 \%$ mortality rate.

Bedside echo or emergency CTPA helps categorize and assess risk based on evidence of RV enlargement/RV dysfunction and make the alternative diagnosis. It would be preferable to start low molecular weight heparin (LMWH) in patients with suspected high-risk PE to avoid delay in starting therapy while carrying routine investigations. LMWH or Fondaparinux (Table 4) is recommended (preferred over unfractionated heparin (UFH)) for most patients as the anticoagulation is mandatory to all. Patients When oral anticoagulation is started, in a patient with PE who is eligible for a NOAC (Apixaban, Dabigatran, Edoxaban, or Rivaroxaban), a NOAC is recommended in preference to a Vitamin K Antagonist (VKA).

Table 4 of LMWH and oral anticoagulation.

VKA anticoagulation initiation should be overlapped with parenteral anticoagulation until an INR of 2.5 (range 2.0 - 3.0) is reached. NOACs should be avoided in patients with severe renal impairment, during pregnancy or lactation, and in patients with antiphospholipid antibody syndrome.

\section{Management of PE}

High-risk or massive PE seen in 5\% of patients presenting with acute PE is

Table 4. Recommended LMWH and oral anti-coagulant doses based European society of cardiology 2019 guidelines.

\begin{tabular}{ccc}
\hline & Dosage & Interval \\
\hline \multirow{2}{*}{ Enoxaparin } & $1.0 \mathrm{mg} / \mathrm{kg}$ & Every $12 \mathrm{~h}$ \\
& Or & \\
Tonzaparin & $1.5 \mathrm{mg} / \mathrm{kg}$ & Once daily \\
& $175 \mathrm{IU} / \mathrm{kg}$ & Once daily \\
& $100 \mathrm{IU} / \mathrm{kg}$ & Every $12 \mathrm{~h}$ \\
Dalteparin & Or & Once daily \\
& $200 \mathrm{IU} / \mathrm{kg}$ & Every $12 \mathrm{~h}$ \\
& $86 \mathrm{IU} / \mathrm{kg}$ & \\
Nadroparin & Or & Once daily \\
& $171 \mathrm{IU} / \mathrm{kg}$ & Once daily \\
& $5.5 \mathrm{mg}$ (body weight $50-100 \mathrm{~kg}) ;$ & \\
\hline
\end{tabular}


associated with at least $15 \%$ risk of in-hospital death, particularly during the first hours after admission (Figure 6). The crucial determining factor in PE management is the presence and severity of RV systolic dysfunction resulting from acute pressure load. These patients should be treated with reperfusion treatment and hemodynamic support. Thrombolytic therapy of PE is well tolerated with

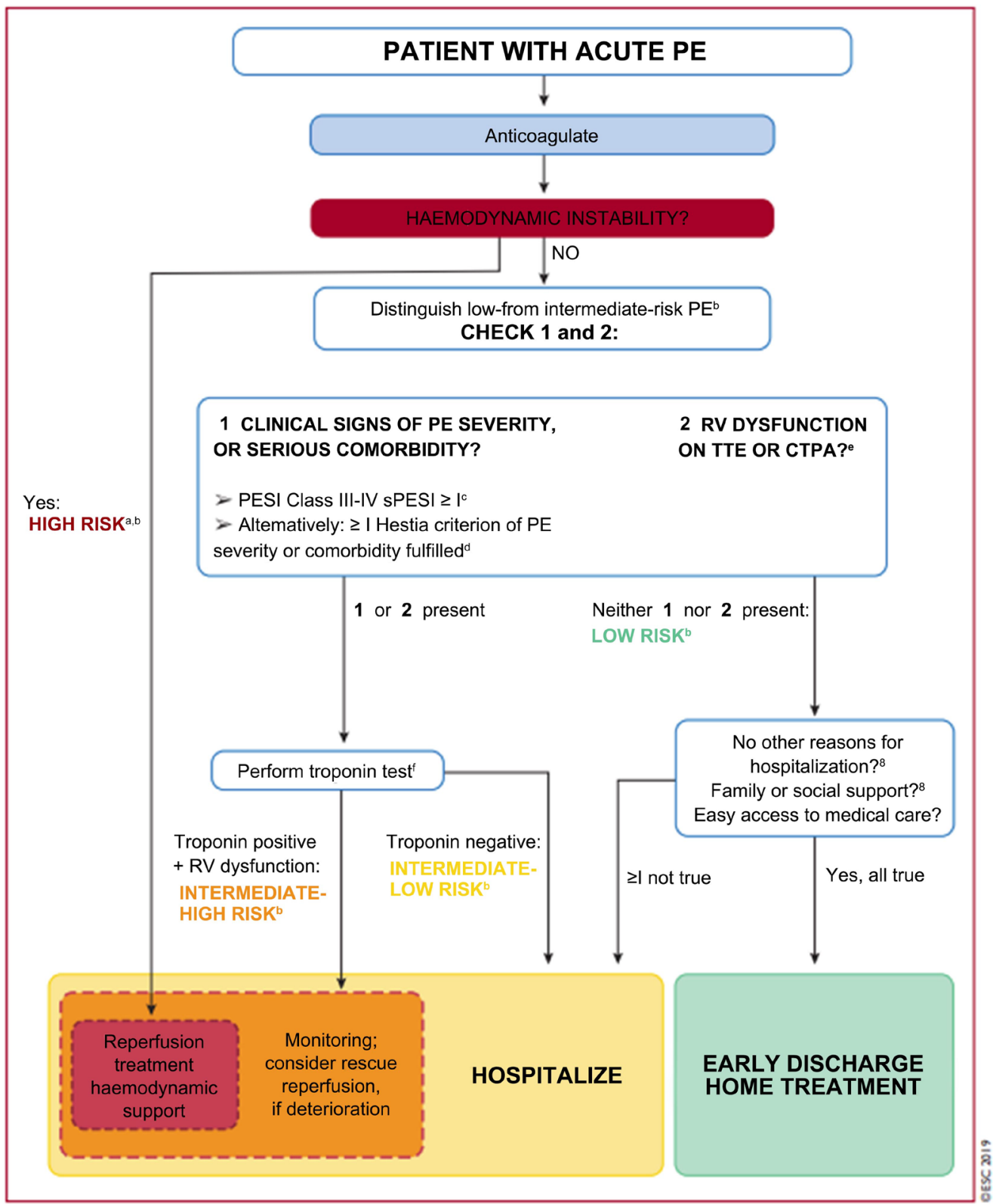

Figure 6. Risk-adjusted management strategy for acute pulmonary embolism (ESC-2019). CTPA = computed tomography pulmonary angiography/angiogram; PE = pulmonary embolism; PESI $=$ Pulmonary Embolism Severity Index; RV = right ventricular; sPESI = simplified Pulmonary Embolism

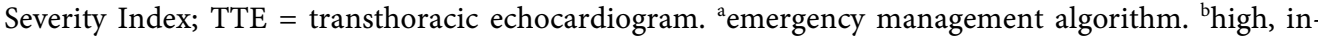
termediate-high-, intermediate-low-, and low-risk PE. 'Cancer, heart failure and chronic lung disease

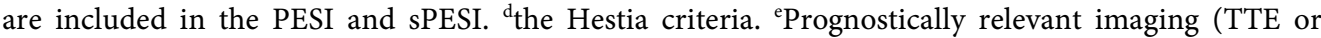
CTPA) findings in patients with acute PE. ${ }^{\mathrm{f}} \mathrm{A}$ cardiac troponin test may already have been performed

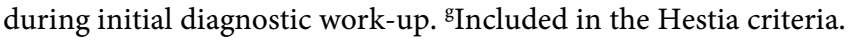


excellent prognosis and carries a risk of significant bleeding 1.8\% - 6.3\% and Intra Cerebral haemorrhage (ICH) $1.2 \%$. It produces faster clot lysing, dissolves obstruction, reverses RV failure, dissolves much of source, and decreases recurrence risk.

\section{Risk-adjusted management strategy for acute PE}

Efficacy and safety of systemic thrombolysis in acute PE well proven in Meta-analysis with the reduction in all-cause mortality, PE mortality, PE recurrence with low bleeding complications. Therapeutic doses and contraindications are given in Table 5.

Plasminogen Activator Italian Multicenter Study II (PAIMS-II) included 36 patients with acute PE treated with rtPA $10 \mathrm{mg}$ bolus followed by $90 \mathrm{mg}$ over 2 hours' period and showed reduced angiographic severity of $\mathrm{PE}$, mean pulmonary artery pressure, and increase of the cardiac index [7]. A randomized, double-blind trial assessing RV function and pulmonary function compared Alteplase Vs Heparin which included 101 stable patients with acute PE showed improvement of $\mathrm{RV}$ function, reduction in RV dimensions, improvement of pulmonary function and no PE occurrence in rtPA group [8]. Another randomized control trial of rtPA $(n=22)$ vs Urokinase $(n=23)$ showed clot lysis in $82 \%$ of rtPA vs $48 \%$ Urokinase treated patients [9]. A European multicenter, double-blind trial showed a decrease in pulmonary vascular resistance at 2 hours, i.e. $18 \% \pm 22 \%$ in Urokinase vs $36 \% \pm 17 \%$ in Alteplase treated patients [10]. RCT of STK vs Alteplase in massive PE with 50 patients showed significant RV ejection fraction and fall of PVR in Alteplase group compared to STK [11].

Table 5. Therapeutic doses and contraindications for acute PE.

\begin{tabular}{|c|c|c|}
\hline Molecule & Regimen & Contraindications to fibrinolysis \\
\hline Recombinant & $100 \mathrm{mg}$ over $2 \mathrm{~h}$ & \multirow{7}{*}{$\begin{array}{l}\text { Absolute: } \\
>\text { History of hemorrhagic stroke or stroke } \\
\text { of unknown origin } \\
>\text { Ischemic stroke in previous } 6 \text { months } \\
>\text { CNS neoplasm } \\
>\text { Major trauma, surgery, or head injury } \\
\text { in previous } 3 \text { weeks } \\
>\text { Bleeding diathesis } \\
>\text { Active bleeding }\end{array}$} \\
\hline $\begin{array}{c}\text { tissue-type } \\
\text { plasminogen (rtPA) }\end{array}$ & $\begin{array}{l}0.6 \mathrm{mg} / \mathrm{kg} \text { over } 15 \mathrm{~min} \\
\text { (maximum dose } 50 \mathrm{mg} \text { ) }\end{array}$ & \\
\hline \multirow{5}{*}{ Streptokinase } & $250,000 \mathrm{IU}$ as a loading over & \\
\hline & $30 \mathrm{~min}$, followed by 100,000 & \\
\hline & $\mathrm{IU} / \mathrm{h}$ over $12-24 \mathrm{~h}$ & \\
\hline & Accelerated regimen: 1.5 & \\
\hline & million IU over $2 \mathrm{~h}$ & \\
\hline \multirow{12}{*}{ Urokinase } & $4400 \mathrm{IU} / \mathrm{kg}$ as a loading dose & \multirow{7}{*}{$\begin{aligned} & \text { Relative: } \\
& \text { Transient ischemic attack in previous } 6 \\
\text { months } & \\
> & \text { Oral anticoagulation } \\
> & \text { Pregnancy or first postpartum week } \\
> & \text { Non-compressible puncture sites } \\
> & \text { Traumatic resuscitation }\end{aligned}$} \\
\hline & over $10 \mathrm{~min}$, followed by & \\
\hline & $4400 \mathrm{IU} / \mathrm{kg} / \mathrm{h}$ over $12-24 \mathrm{~h}$ & \\
\hline & \multirow{9}{*}{$\begin{array}{l}\text { Accelerated regimen: } \\
3 \text { million IU over } 2 \mathrm{~h}\end{array}$} & \\
\hline & & \\
\hline & & \\
\hline & & \\
\hline & & $>$ Refractory hypertension (systolic BP > \\
\hline & & $180 \mathrm{mmHg})$ \\
\hline & & $>$ Advanced liver disease \\
\hline & & $>$ Infective endocarditis \\
\hline & & $>$ Active peptic ulcer \\
\hline
\end{tabular}


All patients with massive PE should be tried with fluid expansion if no significant IVC and RV dilatation followed by IV Nor-epinephrine and IV Dobutamine infusion for hypotension correction inotropy. Volume loading saline or ringer's lactate up to $500 \mathrm{ml}$ over 15 to 30 minutes can be considered in patients with average to low central venous pressure. It sometimes can be over distending the RV, worsen ventricular interdependence and reduce $\mathrm{CO}$. Mechanical ventilation with Positive End Expiratory Pressure (PEEP) is to be avoided as far as possible which may worsen the clinical condition, and try to limit tidal volume plateau pressure due to decreasing venous return. Some high-risk PE patients may get benefited with additional mechanical circulatory support like ECMO along with thrombolysis treatment; catheter-directed PE therapy and surgical pulmonary embolectomy.

Rescue thrombolysis therapy is recommended for patients with hemodynamic deterioration in anticoagulation therapy. Alternatively, these patients can be treated with surgical embolectomy or catheter-directed thrombolysis or interventions percutaneously. Routine treatment is not recommended for intermediate or low-risk PE patients. Percutaneous catheter-directed thrombolysis treatment should be considered for high-risk PE patients whose thrombolytic therapy is contraindicated or has failed. ECMO should be considered in combination with surgical embolectomy or catheter-directed therapy in patients with high-risk PE associated with refractory collapse or cardiac arrest (Figure 7).

No clinical benefit of VA-ECMO in patients with PE unless combined with surgical embolectomy or catheter-directed therapies. Catheter-directed thrombolysis includes CDT, lytic assisted devices-pharmaco mechanical, sonic assisted, Mechanical thrombectomy devices like simple suction \pm clot fragmentation, and large bore aspiration.

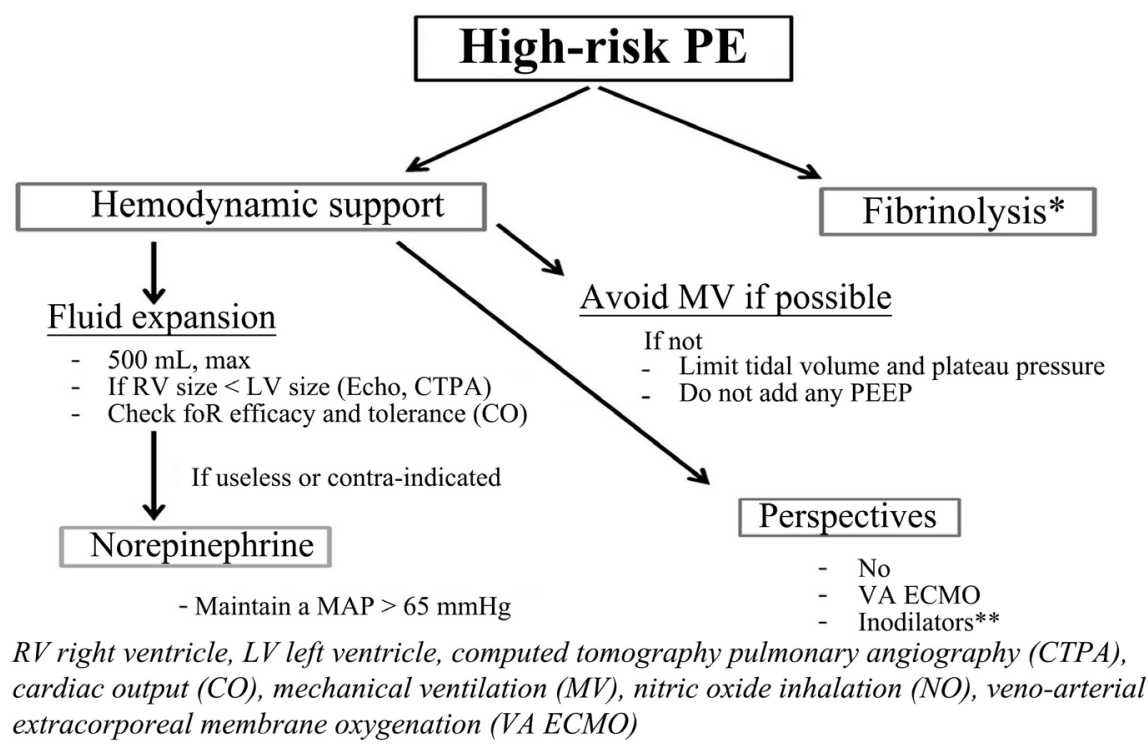

Figure 7. Proposal for hemodynamic management in high-risk PE (Meyer G, Ann. Intensive Care 2016). 
Surgical embolectomy is indicated in patients with massive PE with contraindication to thrombolytic therapy, failed thrombolytic treatment, pregnancy, right heart failure or cardiogenic shock.

European Society of Cardiology (ESC) 2019 guidelines recommended Hestia exclusion criteria for choosing outpatient management of PE (Table 6).

\section{Risk Factors Associated with Recurrent Venous Thromboembolism (VTE) and Anticoagulant-Related Bleeding (Table 7)}

Long-term PE management after discharge depends on the possibility of recurrent

Table 6. Hestia exclusion criteria for choosing outpatient management of PE by ESC 2019.

Criterion/question
Is the patient hemodynamically unstable?
Is thrombolysis or embolectomy necessary?
Active bleeding or high-risk of bleeding?
More than 24 h of oxygen supply to maintain oxygen saturation $>90 \%$ ?
Is PE diagnosed during anticoagulation treatment?
Severe pain needing I.V. pain medication for $>24$ h?
Medical or social reason for treatment in the hospital for $>24 \mathrm{~h}$ (infection, malignancy, no support
system)?
Does the patient have a creatinine clearance $<30 \mathrm{~mL} / \mathrm{min}$ ?
Does the patient have severe liver impairment?
Is the patient pregnant?
Does the patient have a documented history of heparin-induced thrombocytopenia?
If at least one of the questions is answered with "yes", the patient can't discharged early.

Table 7. Risk factors associated with recurrent VTE and anticoagulant-related bleeding.

\begin{tabular}{ccc}
\hline Recurrent VTE & Serious or fatal bleeding & $\begin{array}{c}\text { Both recurrent VTE } \\
\text { and severe bleeding }\end{array}$ \\
Initial unprovoked VTE & Low platelet count & Increased age \\
Initial proximal DVT or PE & Previous bleeding & Cancer \\
Thrombophilia & Recent major bleeding & Immobilization \\
Residual proximal thrombosis & Previous stroke & Recent surgery (transient) \\
Male sex & Hepatic failure & Severe renal impairment \\
Elevated D-dimer concentrations & Diabetes & \\
When not receiving anticoagulation & Abnormal prothrombin time & \\
Pregnancy & Thrombocytopenia & \\
Anticoagulation lasting $<3$ months & Poor anticoagulant control & \\
& Comorbidity & \\
& Anemia & \\
& &
\end{tabular}


PE, fatal bleeding and balancing the risk vs benefit of preventing recurrence vs bleeding related events. Standard anticoagulation therapy for at least three months is recommended to all patients, and it can be stopped after three months for patients with 1st PE/VTE secondary to major transient/reversible risk factor.

Indefinite duration is recommended for recurrent VTE (i.e. with at least one previous PE or DVT) not related to a significant transient or reversible risk factor and patients with antiphospholipid antibody syndrome. It would be preferable to assess drug tolerance and adherence, hepatic and renal function, and the bleeding risk at regular intervals during follow-up. For patients with PE and cancer, weight-adjusted subcutaneous LMWH should be considered for the first six months over VKAs. Edoxaban and Rivaroxaban in patients without gastrointestinal cancer should be considered as an alternative treatment to LMWH. Routine use of IVC filter is not recommended, indicated to PE patients with absolute contraindication to anticoagulation and in case of $\mathrm{PE}$ recurrence despite therapeutic anticoagulation [5].

In a pregnant patient with suspected $\mathrm{PE}$, venous CUS should be considered to avoid unnecessary irradiation. Perfusion scan or CTPA should be considered to rule out suspected PE. LMWH therapy should be considered for all pregnant women with PE, thrombolytic therapy or surgical embolectomy should be recommended for patients with high-risk PE. NOACs should be avoided during pregnancy or lactation period [5].

\section{Pulmonary Embolism Response Team (PERT) Approach}

With the prompt response, PE management with Pulmonary Embolism Response Team (PERT) improves patient outcomes using collaborative, multidisciplinary teams with the best therapeutic options available with well-ordination protocol-based services (Figure 8). It was observed that with PERT based management reduces delay in diagnosis and treatment, reducing mortality by choosing the best possible therapeutic options.

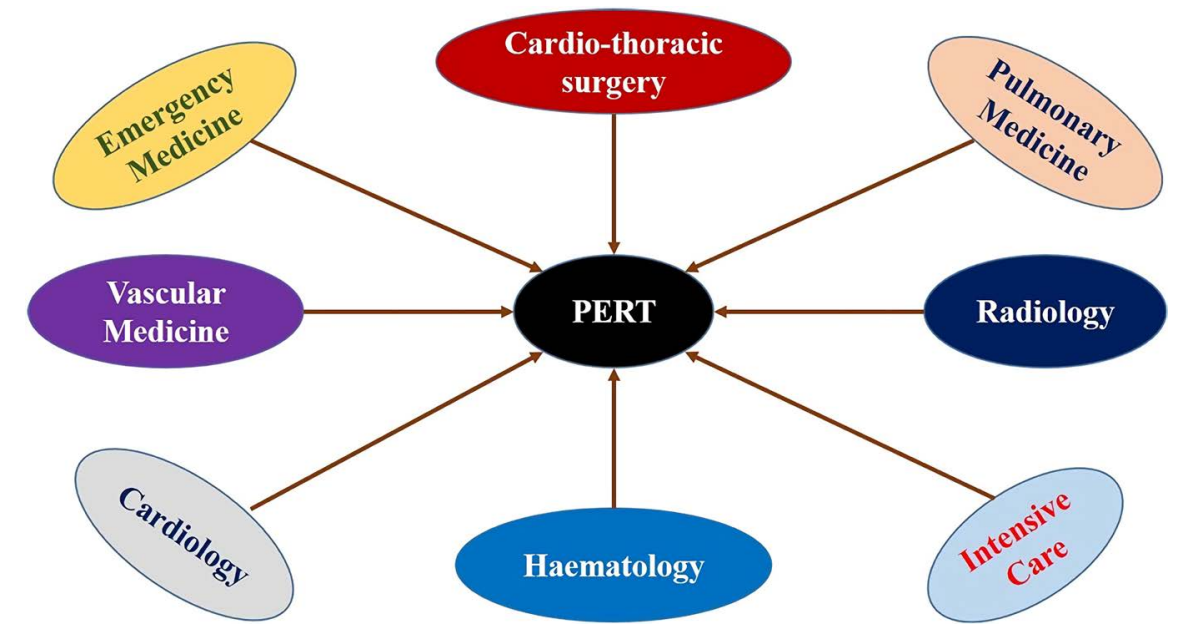

Figure 8. Pulmonary embolism response team. 


\section{Conclusion}

VTE clinically presenting as DVT or PE, is globally the third most frequent acute cardiovascular syndrome behind myocardial infarction and stroke. Thrombolytic therapy is recommended in all patients with high risk PE, unless contraindicated. Routine use of primary systemic thrombolysis is not recommended in patients with intermediate-or low-risk PE. The assembly of a PERT can offer a way to expeditiously and simultaneously engage multiple experts to generate a thoughtful, coordinated, and comprehensive treatment plan for patients with PE.

\section{Conflicts of Interest}

The author declares no conflicts of interest regarding the publication of this paper.

\section{References}

[1] Kyrle, P.A. and Eichinger, S. (2005) Deep Vein Thrombosis. The Lancet, 365, 1163-74. https://doi.org/10.1016/S0140-6736(05)71880-8

[2] Padilla, J.C., Chaparro, P.E., Molina, K., Arevalo-Herrera, M. and Herrera, S. (2015) Is There Malaria Transmission in Urban Settings in Colombia? Malaria Journal, 14, 1-9. https://doi.org/10.1186/s12936-015-0956-0

[3] Joseph, T. (2016) Pulmonary Embolism- Diagnosis. https://www.slideshare.net/drtinkujoseph2010/pulmonary-embolism-diagnosis-bydrtinku-joseph

[4] Meyer, G., Vieillard-Baron, A. and Planquette, B. (2016) Recent Advances in the Management of Pulmonary Embolism: Focus on the Critically Ill Patients. Annals of Intensive Care, 6, 1-8. https://doi.org/10.1186/s13613-016-0122-Z

[5] Stavros, V.K., Guy, M., Cecilia, B., Héctor, B., Geert-Jan, G., Veli-Pekka, H., Menno, V.H., Marc, H., Catriona, S.J., David, J., Nils, K., Irene, M.L., Mareike, L., Roberto, L., Lucia, M., Nicolas, M., Fionnuala, N.Á., Paolo, P., Piotr, P., Marc, R., Adam, T., Eric Van, B., José, L.Z., ESC Scientific Document Group (2020) 2019 ESC Guidelines for the Diagnosis and Management of Acute Pulmonary Embolism Developed in Collaboration with the European Respiratory Society (ERS): The Task Force for the Diagnosis and Management of Acute Pulmonary Embolism of the European Society of Cardiology (ESC). European Heart Journal, 41, 543-603

[6] Van der Meer, R.W., Pattynama, P.M., van Strijen, M.J., van den Berg-Huijsmans, A.A., Hartmann, I.J., Putter, H., de Roos, A. and Huisman, M.V. (2005) Right Ventricular Dysfunction and Pulmonary Obstruction Index at Helical CT: Prediction of Clinical Outcome during 3-Month Follow-up in Patients with Acute Pulmonary Embolism. Radiology, 235, 798-803. https://doi.org/10.1148/radiol.2353040593

[7] Dalla-Volta, S., Palla, A., Santolicandro, A., Giuntini, C., Pengo, V., Visioli, O., Zonzin, P., Zanuttini, D., Barbaresi, F., Agnelli, G. and Morpurgo, M. (1992) PAIMS 2: Alteplase Combined with Heparin Versus Heparin in the Treatment of Acute Pulmonary Embolism. Plasminogen Activator Italian Multicenter Study 2. Journal of the American College of Cardiology, 20, 520-526. https://doi.org/10.1016/0735-1097(92)90002-5

[8] Goldhaber, S.Z., Come, P.C., Lee, R,T., Braunwald, E., Parke,r J.A., Haire, W.D., 
Feldstein, M.L., Miller, M., Toltzis, R., Smith, J.L. and de Silva, A.T. (1993) Alteplase Versus Heparin in Acute Pulmonary Embolism: Randomised Trial Assessing Right-Ventricular Function and Pulmonary Perfusion. The Lancet, 341, 507-511. https://doi.org/10.1016/0140-6736(93)90274-K

[9] Goldhaber, S., Heit, J., Sharma, G.V., Nagel, J.S., Kim, D., Parker, J.A., Drum, D., Reagan, K., Anderson, J., Kessler, C. and Markis, J. (1988) Randomised Controlled Trial of Recombinant Tissue Plasminogen Activator Versus Urokinase in the Treatment of Acute Pulmonary Embolism. The Lancet, 332, 293-298.

https://doi.org/10.1016/S0140-6736(88)92354-9

[10] Meyer, G., Sors, H., Charbonnier, B., Kasper, W., Bassand, J.P., Kerr, I.H., Lesaffre, E., Vanhove, P. and Verstraete, M. (1992) Effects of Intravenous Urokinase Versus Alteplase on Total Pulmonary Resistance in Acute Massive Pulmonary Embolism: A European Multicenter Double-Blind Trial. Journal of the American College of Cardiology, 19, 239-245. https://doi.org/10.1016/0735-1097(92)90472-Y

[11] Meneveau, N., Schiele, F., Vuillemenot, A., Valette, B., Grollier, G., Bernard, Y. and Bassand, J.P. (1997) Streptokinase vs Alteplase in Massive Pulmonary Embolism: A Randomized Trial Assessing Right Heart Haemodynamics and Pulmonary Vascular Obstruction. European Heart Journal, 18, 1141-1148.

https://doi.org/10.1093/oxfordjournals.eurheartj.a015410 